\title{
Testing gravity theories in the radiative regime using pulsar timing arrays
}

\author{
K. J. Lee \\ Max-Planck-Institut für Radioastronomie, Bonn 53121, Germany \\ email: kjlee007@gmail.com
}

\begin{abstract}
General relativity has predicted the existence of gravitational waves (GW), which are waves of the distortions of space-time with two degrees of polarization and the propagation speed of light. Alternative theories predict more polarizations, up to a maximum of six, and possible frequency dependent propagation speed from the light speed. The polarization and dispersion properties of GWs shed light on the spin and mass information of gravitons. Although GWs have not been directly detected yet, their amplitude upper-bounds has been addressed by research using different types of detectors. For example, the amplitude upper-bounds for the stochastic background derived from pulsar timing observations have already become astrophysically interesting. The present paper reviews proposals to test the gravity theories in the radiation regime by observing GWs using pulsar timing arrays. We also present the estimation for the upper-bounds on the amplitude of alternative modes for the stochastic background of GW.
\end{abstract}

Keywords. gravitational waves, (stars:) pulsars: general, elementary particles

\section{Introduction}

Two major characteristics of GWs are important to differentiate the validity of gravity theories in the radiative regime; the polarization and dispersion of $\mathrm{GW}$ in vacuum. In alternative metric theories, GW can have up to six possible polarization states, four more than which are allowed by GR. Furthermore, the propagation speed of GW can deviate from the predication of GR that GW propagates at light speed in vacuum, i.e. the effective graviton mass is zero.

A pulsar timing array is a unique technique to detect nano-Hertz GWs by timing multiple millisecond pulsars, which are very stable celestial clocks (Jenet et al. 2005). It turns out that a stochastic GW background leaves an angular-dependent correlation in pulsar timing residuals for widely spaced pulsars (Hellings \& Downs, 1983): the correlation $C(\theta)$ between timing residual of pulsar pairs is a function of angular separation $\theta$ between the pulsars. One can analyse the timing residual and measure such a correlation to detect GWs (Jenet et al. 2005). Lee et al. (2008, 2010) have found that the exact form of $C(\theta)$ is very different from the one of GR, if the GW has extra polarization state or graviton mass is not zero. By measuring the correlation function, we can directly test gravity theories in the radiative regime.

\section{Pulsar timing correlation functions and gravity tests}

A GW introduces extra signal in pulsar timing data. Let the unit vector of the GW propagation direction be $\hat{\mathbf{e}}_{z}, \mathrm{GW}$ frequency be $f$, the direction from the observer to the photon source (pulsar) be $\hat{\mathbf{n}}^{i}$. The GW induced frequency-shift of a pulsar timing signal 
is (Lee et al. 2008, 2010; Baskaran et al. 2008)

$$
\frac{\Delta \omega(t)}{\omega}=\frac{\hat{\mathbf{n}}^{i} \hat{\mathbf{n}}^{j}}{2\left(1+\frac{c}{2 \pi f} \mathbf{k}_{\mathrm{g}} \cdot \hat{\mathbf{n}}\right)}\left[h_{i j}(t, 0)-h_{i j}(t-|\mathbf{D}| / \mathbf{c}, \mathbf{D})\right],
$$

where the $\mathbf{D}$ is the displacement vector from the observer to the pulsar, $h_{i j}(t, 0)$ and $h_{i j}(t-|\mathbf{D}| / \mathbf{c}, \mathbf{D})$ are the metric perturbations by GW at the Earth and at the pulsar when the received pulse was emitted, $\omega$ is the angular frequency for the pulsar pulse, $f_{\text {cut }}=m_{\mathrm{g}} c^{2} / h$ is the cut-off frequency of GW due to the graviton mass $m_{\mathrm{g}}$, and the $k_{\mathrm{g}}$ is the GW wave vector given by Lee et al. (2010)

$$
\mathbf{k}_{\mathrm{g}}(f)=\frac{2 \pi\left(f^{2}-f_{\text {cut }}^{2}\right)^{\frac{1}{2}}}{c} \hat{\mathbf{e}}_{z} .
$$

The induced pulsar timing residuals $R(t)$ are given by the temporal integration of above the frequency shift at Earth given above, thus $R(t)=\int_{0}^{t} \frac{\Delta \omega(\tau)}{\omega} d \tau$.

The spatial metric perturbation $h_{i j}(t, \mathbf{r})$ induced by a stochastic GW background is a superposition of monochromatic GWs with random phases and amplitudes. It is (Maggiore, 2000)

$$
h_{i j}\left(t, r^{i}\right)=\sum_{P=+, \times} \int_{-\infty}^{\infty} d f \int d \Omega h^{P}\left(f, \hat{\mathbf{e}}_{z}\right) \epsilon_{i j}^{P}\left(\hat{\mathbf{e}}_{z}\right) e^{i\left[2 \pi f t-k_{\mathrm{g}}{ }^{i}(f) r_{i}\right]},
$$

where $\Omega$ is the solid angle, index $i, j$ run from 1 to $3, h^{P}$ is the amplitude of the GW propagating in the direction of $\hat{\mathbf{e}}_{z}$ per unit solid angle per frequency of polarization state $P$, and the polarization tensor $\epsilon_{a b}^{P}$ of GWs are given in details in Lee et al. (2008). The superscript $P$ takes value of ',$+ \times$ ' for the two Einsteinian modes of GW polarization, ' $b$ ' and ' $l$ ' for the breathing and the longitudinal mode respectively, and ' $s n, s e$ ' for the shear modes.

Such stochastic GW background leaves a correlation between timing residuals of pulsars pairs. Such correlation, $C(\theta)$, depends on the angular distance $\theta$ between two pulsars as well as on the polarization of GW and graviton mass.

Lee et al. (2008) have calculated the pulsar timing correlation function for all the polarization modes of GW. For the Einsteinian modes and for the breathing mode, the cross-correlation function $C^{P}(\theta)$ is independent of earth-pulsar distances and independent of the GW characteristic strain spectrum. In contrast, for the modes that are not purely transverse, the shear and longitudinal modes, the cross correlation functions depend on the specifics of the strain spectra and on the pulsar distribution in distance.

Fig. 1 shows the correlation function according to different classes of GW polarization. Clearly by comparison of these 'theoretical' correlation curves with observations we can test the polarization state of GWs.

Lee et al. (2010) have calculated the pulsar timing correlation function for a GW background with none-zero-mass graviton. They noted that the pulsar timing crosscorrelation function for a massive GW background depends on the graviton mass, specific power spectra of the GW background, and on the observation schedule. The 5-year and 10-year correlation functions are reproduced in Fig. 2, where the graviton with the same mass introduces more deviation to the 10-year correlation function than it does to the 5-year one.

Intuitively speaking, the necessary conditions for a positive detection of a graviton mass should be: 1 . The GW is strong enough such that the GW can be detected; 2 . the physical effects of alternative theories should be strong enough to see the deviation from 


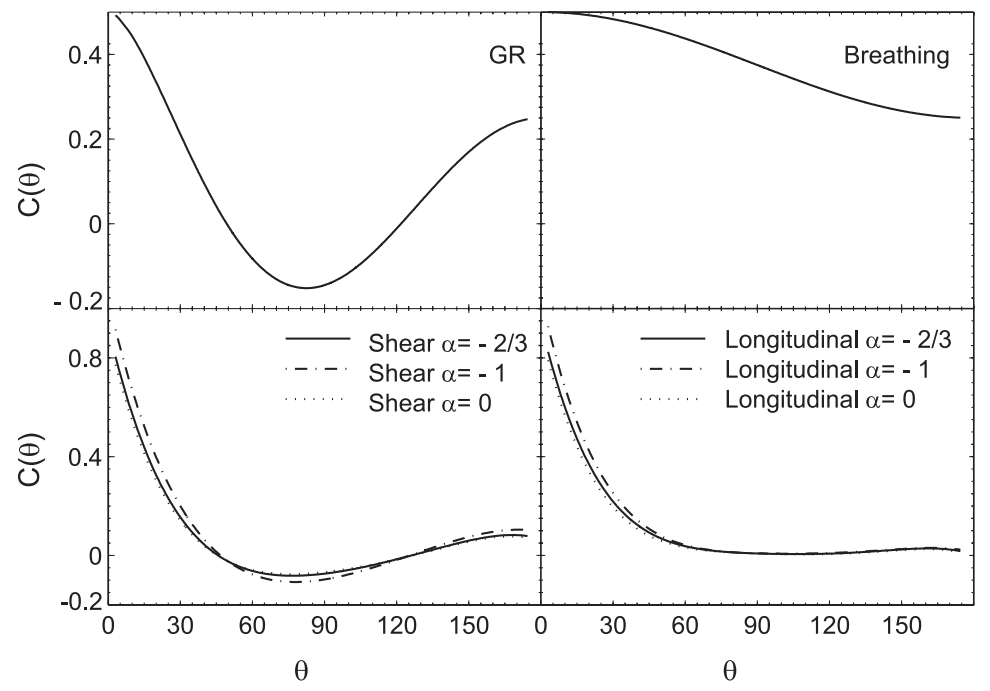

Figure 1. The normalized pulsar timing residual correlation coefficients. Here $\theta$ is the angular separation between two pulsars. 'GR' stands for the two transverse traceless modes, ' + ' and ' $x$ '. Results are given for several values of $\alpha$, the power-law index of the GW spectrum.

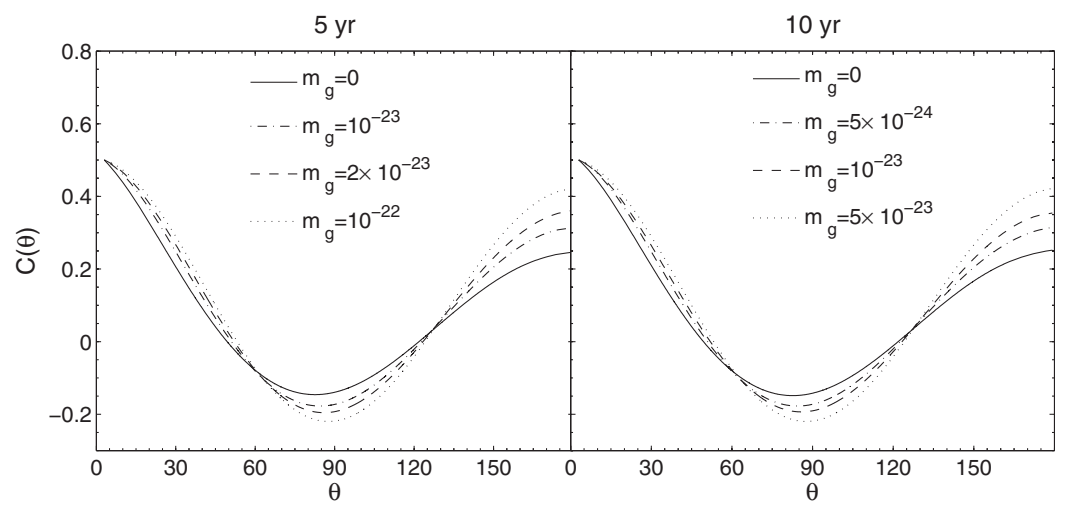

Figure 2. The atlas for cross-correlation functions $C(\theta)$. The label of each curve indicates the corresponding graviton mass in unit of electron-volts $(\mathrm{eV})$. The left panel are the correlation functions for a 5-year bi-weekly observations. The right panel shows correlation functions for 10 -year bi-weekly cases. We take $\alpha=-2 / 3$ for these results. These correlation are normalized such that the $C(0)=0.5$ for two different pulsars.

GR. These intuition is confirmed by simulations in, which show that the high detection rate is achieved only if one has enough pulsar and if the graviton mass is large enough or if GW of alternative polarization modes is strong enough.

For identifying the polarization modes, observation shows that if bi-weekly observations are made for five years with RMS timing accuracy of $100 \mathrm{~ns}$, then 60 pulsars are required for the longitudinal mode; 60 for the two spin-1 "shear" modes; and 40 for the spin 0 "breathing" mode and 40 pulsars are needed for the detection of the GR modes.

For detecting massive graviton, simulations have shown that we need at least 60 pulsars to be able to tell the difference between a massive GW background and a massless one. For 5-year timing of 100 pulsar we can start to detect a graviton heavier than $2.5 \times 10^{-22}$ $\mathrm{eV}$ and we can achieve a limit of $m_{\mathrm{g}}=10^{-22} \mathrm{eV}$ by using 5 -year observation of 300 
pulsars. We can achieve levels of $10^{-22} \mathrm{eV}$ and $5 \times 10^{-23} \mathrm{eV}$ in a 10 -year observation using 100 and 300 pulsars respectively.

\section{Estimation for the upper-bounds on the amplitude of the alternative modes}

The power spectra $S(f)$ of the timing residual is defined as $S(f)=2 \int_{-\infty}^{\infty} d t e^{-2 \pi i f t}$ $\left\langle R\left(t^{\prime}\right) R\left(t^{\prime}+t\right)\right\rangle$, with $f \geqslant 0$. Assuming an power-law spectra for the stochastic background of GW, i.e. characteristic strain $h_{\mathrm{c}} \equiv A_{c}\left(f / f_{\mathrm{c}}\right)^{\alpha}$, one can show that

$$
\begin{aligned}
S_{\mathrm{R}}^{+, \times}(f) & =\frac{A_{c}^{2} f^{2 \alpha-3}}{24 \pi^{2} f_{c}^{2 \alpha}} \\
S_{\mathrm{R}}^{\mathrm{b}}(f) & =\frac{A_{c}^{2} f^{2 \alpha-3}}{12 \pi^{2} f_{c}^{2 \alpha}} \\
S_{R}^{\mathrm{sn}, \mathrm{se}}(f) & =\frac{A_{c}^{2}\left(3 \ln (4 \pi f D / c)+3 \gamma_{e}-7\right) f^{2 \alpha-3}}{24 \pi^{2} f_{c}^{2 \alpha}} \\
S_{\mathrm{R}}^{\mathrm{l}}(f) & =\frac{A_{c}^{2} D f^{2 \alpha-2}}{16 c f_{c}^{2 \alpha}},
\end{aligned}
$$

where the $D$ is the distance of the pulsar, $\gamma_{e} \simeq 0.58$ is the Euler's $\gamma$ constant. The superscripts take the same meaning as in the polarization index ' $P$ ' in the $h^{P}$. One can see that the power spectrum of the longitudinal mode and shear modes are proportional to the distance of pulsar $D$ and its logarithm $\ln D$ respectively. The physical reasons for the phenomena is that the GWs of the two modes and pulsar signals traveled and kept the phases along the similar path, in this way, the pulsar signals could accumulate the GW effects. Recently, this has also been noted by Alves \& Tinto (2011) and Chamberlin \& Siemens (2012).

These formulae can be used to "estimate" the upper-bounds for the alternative modes by converting the results from upper-bounds for the GR modes. From Equations(3.1), we can see that the spectra of the timing residuals are all power-law like for power-law GW background. In this way, we can translate the upper-bounds as function of power index $\alpha$ for GR modes to the alternative modes as

$$
\begin{aligned}
A_{\mathrm{up},}^{(\mathrm{b})}(\alpha) & =A_{\mathrm{up}}^{(\mathrm{GR})}(\alpha) \\
A_{\mathrm{up}}^{(\text {shear })}(\alpha) & =\frac{A_{\mathrm{up}}^{(\mathrm{GR})}(\alpha)}{\sqrt{3 \ln \left(4 \pi f_{0} D / c\right)+3 \gamma_{e}-7}} \\
A_{\mathrm{up}}^{(\mathrm{l})}(\alpha) & =\frac{A_{\mathrm{up}}^{(\mathrm{GR})}\left(\alpha+\frac{1}{2}\right)}{\pi} \sqrt{\frac{4 c}{3 D f_{c}}} .
\end{aligned}
$$

Using the upper bounds derive by Jenet et al. (2006), we can estimate the upper bounds for alternative modes, as given in the Figure. (3)

\section{Conclusion and Discussion}

The stochastic GW background produces extra timing signals in pulsar TOA data, and one can detect the GW background by precise timing several pulsars and measuring the angular dependent correlations between the timing signals of several pulsars. A precise measurement of the angular correlation function can, in principle, determine the GW polarization properties of the GWs making up the stochastic background as well as 


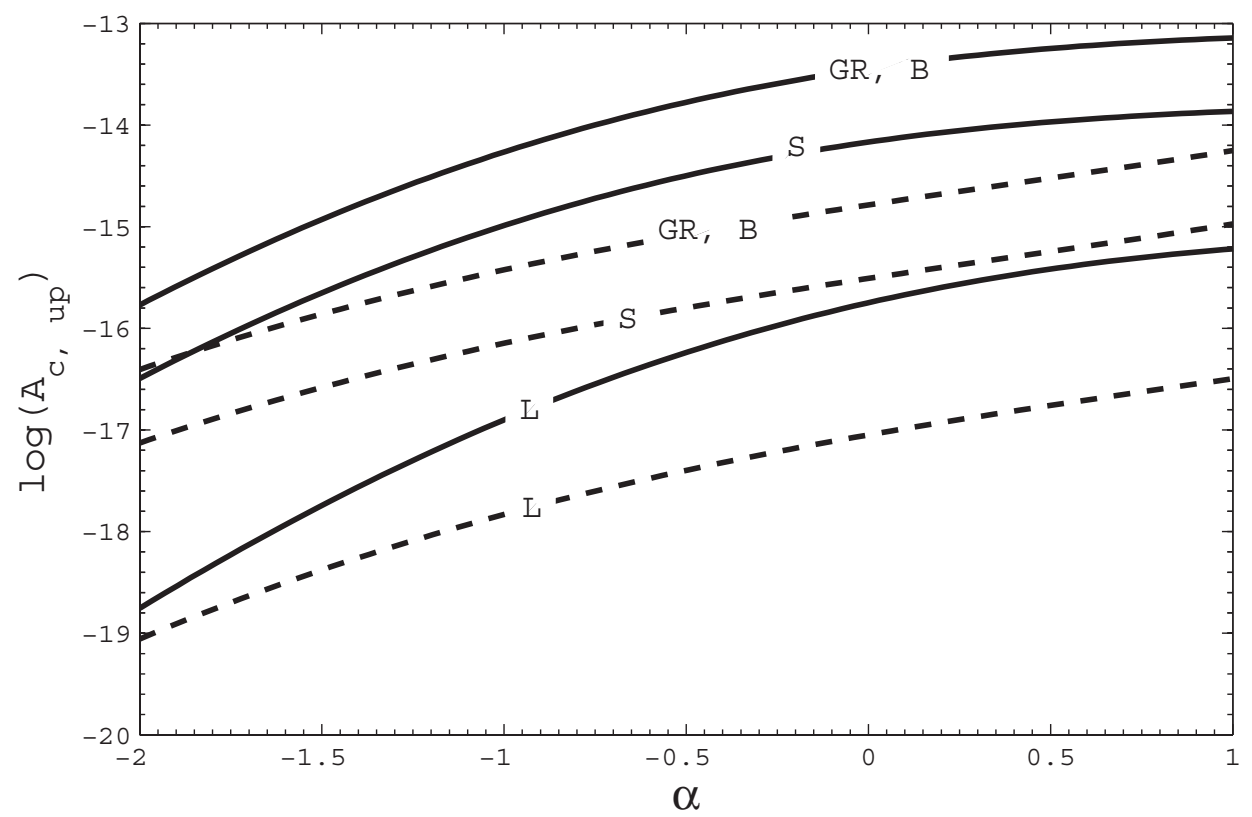

Figure 3. The upper-bounds for the characteristic strain of stochastic GW background of GR and alternative polarization modes. The solid lines correspond to the current upper-bounds, while the dashed lines are the potential upper-bounds given 5 year observing for 20 pulsars with 100 ns intrinsic pulsar timing noise. On each line, the label 'GR, B, S, L' stand for the upper-bounds for GR, breathing, shear, and longitudinal modes respectively. We have used a 5 th order polynomial to make curves smooth compared with results of Jenet et al. (2006).

the graviton mass. A large number of pulsars with good timing precise of $\sim 100 \mathrm{~ns}$ are required to successfully perform tests for gravity theories in radiative regime. In this regard, pulsar surveys' success in finding more millisecond pulsars is critical. To time such large number of pulsars, the Large European Array for Pulsars (Stappers, Vlemmings, \& Kramer, 2009), the Five-hundred-meter Aperture Spherical Radio Telescope (Nan et al., 2006, Smits et al., 2009) and the Square Kilometer Array (SKA) will offer unique opportunities to detect the GW background and measure its properties.

\section{Acknowledgment}

K.J.Lee gratefully acknowledges support from ERC Advanced Grant "LEAP" Grant Agreement Number 227947 (PI: M. Kramer).

\section{References}

M. E. D. S. Alves \& M. Tinto, 2011 Phys. Rev. D 838313529.

S. J. Chamberlin \& X. Siemens, 2012 Phys. Rev. D 85082001.

F. A. Jenet, G. B. Hobbs, K. J. Lee, \& R. N. Manchester, 2005 ApJL 625, L123-L126.

F. A. Jenet, et al., 2006 ApJ, 653, 1571.

R. W. Hellings \& G. S. Downs, 1983 ApJL 265, L39-L42.

K. J. Lee, F. A. Jenet, \& R. H. Price, 2008 ApJ 685, 1304-1319.

K. Lee, F. A. Jenet, R. H. Price, N. Wex, \& M. Kramer, 2010 ApJ 722, 1589-1597.

D. Baskaran, A. G. Polnarev, M. S. Pshirkov, \& K. A. Postnov, 2008 PRD 78, 044018-+.

M. Maggiore, 2000 Phys. Rep. 331, 283-367. 
B. Stappers, W. Vlemmings, \& M. Kramer, 2009 "Pulsars, e-VLBI, \& LEAP (invited)," in Proceedings of the 8th International e-VLBI Workshop. 22-26 June 2009 . Madrid, Spain, p. 20.

R. Nan, Q. Wang, L. Zhu, W. Zhu, C. Jin, \& H. Gan, 2006 Chinese Journal of Astronomy 8 Astrophysics Supplement 6, 020000-310.

R. Smits, D. R. Lorimer, M. Kramer, R. Manchester, B. Stappers, C. J. Jin, R. D. Nan \& D. Li, $2009 A \mathscr{E} A$ 505, 919-926. 